\title{
The Assessment of a Novel Lower Body Resistance Garment As a Mechanism to Increase the Training Stimulus During Running: a Randomised Cross Over Study
}

\section{Samantha M Hoffmann ( $\square$ s.hoffmann@deakin.edu.au )}

Centre for Sport Research (CSR), School of Exercise and Nutrition Sciences, Deakin University, Geelong, VIC, Australia

Isaiah Di Domenico

Centre for Sport Research (CSR), School of Engineering, Deakin University, Geelong, VIC, Australia

\section{Paul K Collins}

Centre for Sport Research (CSR), School of Engineering, Deakin University, Geelong, VIC, Australia

\section{Research Article}

Keywords: wearable resistance, limb loading, exercise physiology

Posted Date: October 6th, 2021

DOl: https://doi.org/10.21203/rs.3.rs-954642/v1

License: (c) (i) This work is licensed under a Creative Commons Attribution 4.0 International License. Read Full License

Version of Record: A version of this preprint was published at BMC Sports Science, Medicine and Rehabilitation on April 8th, 2022. See the published version at https://doi.org/10.1186/s13102-02200455-9. 


\section{Abstract}

Background: This study examined the physiological and perceived impact of wearing a novel lower body resistance garment during exercise and recovery.

Methods: Using a randomised cross over design, 15 recreationally-active males performed 2x10-minute steady-state runs followed by a 10-minute passive recovery with concomitant monitoring of oxygen consumption $\left(\mathrm{VO}_{2}\right)$, heart rate ( $\left.\mathrm{HR}\right)$ and rating of perceived exertion (RPE; exercise portion only), wearing either the resistance garment (experimental) or running shorts (control).

Results: During exercise, $\mathrm{VO}_{2}$ and RPE were higher ( $4.5 \%$ and $7.7 \%$ respectively) in experimental than control $\left(\mathrm{VO}_{2}: r=0.24, p>0.05\right.$; RPE: $\left.r=0.32, p>0.05\right)$ whilst HR was lower $(-0.4 \%, r=-0.05, p>0.05)$. During recovery, $\mathrm{VO}_{2}$ and $\mathrm{HR}$ were lower ( $4.7 \%$ and $4.3 \%$ respectively) in experimental than control $\left(\mathrm{VO}_{2}: r=-0.32\right.$, $p>0.05$; HR: $r=-0.27, p>0.05)$.

Conclusions: Though effects were trivial to small, and not statistically significant, these findings provide proof of concept and suggest that this garment design may increase the training stimulus during running and aid post-exercise recovery.

\section{Background}

Progressive overload ensures health and performance benefits are achievable and sustainable $(1,2)$. There are many ways to apply progressive overload in exercise training with recreational and athletic populations (3). Wearable resistance (WR) is a method whereby external loading is placed upon the body during exercise with the goal of increasing movement difficulty, therefore providing a greater training stimulus - all without causing undesirable movement patterns $(4,5)$. A variety of WR loading orientations have been examined including trunk loading via weighted vests $(6,7)$, weighted pulley systems (6) and loaded back packs (8), and lower limb loading via thigh and foot weights sewn into clothing (9) and weighted ankle bands (10). During exercise at sub-maximal intensities $\left(6.4-14.4 \mathrm{~km} \mathrm{~h}^{-1}\right)$, these loading orientations have shown considerable increases in oxygen consumption $\left(\mathrm{VO}_{2}\right)(6,7,9,10)$, heart rate (HR) (7-10), and perceived exertion (8) during exercise. However, negative side effects including altered running mechanics have been reported when weighted vests were worn (6) and discomfort has been perceived with the use of weighted ankle bands (10) and loaded back packs (8).

Previous research has examined varying magnitudes of applied resistance in WR apparel on both acute and chronic responses to exercise. Low levels of resistance (1-5\% body mass; BM) in the form of compression shorts increased $\mathrm{HR}(0.4-2.9 \%)$ and $\mathrm{VO}_{2}(1.7-8.1 \%)$ in endurance athletes during 8 minutes of steady-state running (11). Conversely, with higher levels of resistance (5-10\% BM) in the form of weighted vests, endurance athletes experienced no significant increase in $\mathrm{VO}_{2}$ during 12 minutes of steady-state running compared to control (6), and no significant increase in maximal oxygen consumption $\left(\mathrm{VO}_{2} \mathrm{max}\right)$ following four weeks of WR training when compared to control (12). However, 
with a resistance of $10-20 \%$ BM using weighted vests, a significant increase in $\mathrm{VO}_{2}$ (10.8-16.8\%) was shown in recreationally-active participants across all stages of an incremental walking test (2.0-4.0 mph) (7). Further increases in resistance $(20 \%, 30 \%$ and $40 \%$ BM) using weighted back packs also showed significant increases in HR (6.3-8.0\%) and perceived exertion (16.7-40.7\%) in recreationally-active participants during an $8 \mathrm{~km}$ walk at a self-selected pace (8). These findings suggest that training status and/ or the magnitude of applied resistance may influence the capacity of WR to increase the physiological and perceived demand of exercise. As such, no common consensus has been reached regarding the optimal magnitude of applied resistance needed to sufficiently increase the physiological demand of exercise without inducing undesirable changes in movement patterns (4).

Recent research has focused on tightly fitted lower limb compression garments as a means of applying WR that is more comfortable for the wearer whilst avoiding undesirable impacts on movement mechanics. These designs include international standard compression materials that conform to the body's natural shape ensuring comfort and avoiding range of motion restrictions (13). The garments provide low levels of loading (1-5\% BM) to the thigh via small, weighted pouches $(100 \mathrm{~g}-200 \mathrm{~g})$ attached to the garment at distal to proximal orientations (11). Although the garment increased $\mathrm{VO}_{2}, \mathrm{HR}$ and perceived exertion during running, the weighted pouches were bulky and required participants to physically manipulate the garment to meet their desired level of resistance. Further research is warranted to explore more practical and effective ways in which WR can be used to increase training stimulus. No previous studies have reported the effect of WR on post-exercise recovery. This may be of practical significance if WR garments exhibit compression qualities. Despite inconclusive effects of compression garments on recovery indicators, there is some evidence for local blood flow augmentation (14), and therefore assessing WR during recovery is warranted. The aim of this study was to examine the physiological and perceptual responses to steady-state running and post-running recovery whilst wearing a novel lower body WR garment with 1-3\% BM applied resistance compared to standard running shorts, in recreational exercisers.

\section{Methods}

\subsection{Subjects}

Subjects were recruited via convenience sampling through word of mouth and email advertisements sent to staff of the Health and Engineering faculties at Deakin University and to players from local amateur football clubs. Inclusion criteria were (a) males aged 18-35 years (inclusive), (b) free of injury and illness, (c) body mass index (BMI) $<30 \mathrm{~kg} \cdot \mathrm{m}^{2}$, (d) recreationally-active, defined as having completed a minimum of 60 minutes of moderate to vigorous intensity physical activity per week for the last six months. Fifteen recreationally-active men (mean \pm SD, age: $24.5 \pm 3.5$ yrs; body mass: $75.5 \pm 8.0 \mathrm{~kg}$; height: $179.9 \pm 8.8$ $\mathrm{cm}$ ) volunteered to participate. Ethics approval was obtained from the local Institutional Human Research Ethics Committee prior to participant recruitment. All subjects provided written informed consent and 
completed the Exercise and Sport Science Australia, Adult Pre-Exercise Screening System (ESSA-APSS) before participating.

\subsection{Procedures}

The present study was conducted in two phases: (1) the design of the garment, and (2) human exercise trials.

\subsubsection{Design Phase}

The novel lower body resistance garment used in this study was comprised of 92\% polyamide and $~ 8 \%$ elastane wherein resistive bands were seamlessly and integrally formed in the fabric of the garment through 3D knitting techniques. As shown in Figure 1, the ' $X$ ' shaped configurations applied 1-3\% BM resistance to the wearer across various anatomical locations (15). These included the hips and knees, and the musculature acting upon these joints including the quadriceps, hamstrings, glutes and lower leg muscles. Due to the general design and materials used, the garment was intended to have a similar look and feel to a conventional compression garment that conformed smoothly to individual body shapes, ensuring comfort for the wearer during movement.

The design was partially developed by use of computer software (Abaqus V6.14) to investigate placement of the resistance-based zones as well as indicative elastic moduli of the knitted base materials. A Finite Element Analysis (FEA) model was created, based off a male in the 75th percentile for height $(\sim 178 \mathrm{~cm})$ and weight $(\sim 75 \mathrm{~kg})$. The FEA model compared joint reaction moments from the hip and knee and when compared to a non-WR garment. It was noted that $11.5 \%$ and $8.9 \%$ increases in reaction moments occurred at the hip and knee, respectively. Garment samples were produced and subsequently deconstructed with material samples tested on a $10 \mathrm{kN}$ Instron load frame showing a difference in elastic modulus of the banded $(6.98 \pm 0.82 \mathrm{MPa})$ versus non-branded materials $(3.13 \pm 0.21$ $\mathrm{MPa})$. The FEA simulation in conjunction with material data, determined that the WR garment provided greater theoretical resistance against hip and knee rotation as compared to a traditional non-WR garment.

\subsubsection{Exercise Phase}

A cross-over study design was used whereby subjects reported to the Exercise Science Laboratory on two separate occasions separated by an average of 5 days. In a randomised order, subjects completed a steady-state running trial, once on each occasion, wearing either the novel lower body resistance garment ('experimental trial') or a pair of standard exercise shorts of their choosing ('control trial'). Each subject completed their trials at a similar time of day under stable laboratory conditions (mean \pm SD, temperature: $17.7 \pm 1.2^{\circ} \mathrm{C}$; humidity: $43.3 \pm 2.1 \%$ ) on a motorized treadmill (150/50 LC, HP-Cosmos $\rightarrow$, NussdorfTrainstein, Germany) at a gradient of $1 \%$ to reflect outdoor running demands (16). Treadmill running speed was constant and set to one of two levels; 'low' $\left(9.8 \mathrm{~km} \mathrm{~h}^{-1}\right)$ or 'high' (11.9 $\left.\mathrm{km} \mathrm{h}^{-1}\right)$, to account for cardiorespiratory fitness differences across subjects. Subjects who completed $<150$ minutes of moderate-intensity physical activity per week were assigned to the 'low' running speed $(n=\lambda)$ and those who completed > 150 minutes of moderate-intensity physical activity per week were assigned the 'high' 
running speed $(n=8)$. Both running speeds aimed to achieve an exercise intensity of $60 \% \mathrm{VO}_{2}$ max using the following equation: $\mathrm{VO}_{2}=13.5 \times$ speed -8.5 ; a published equation for the relationship between $\mathrm{VO}_{2}$ and running speed (16). $\mathrm{VO}_{2}$ max for those classified as 'low' was based on normative data provided by the American College of Sports Medicine (ACSM) (17) for 20-29-year-old males (47 ml kg-1 $\left.\mathrm{min}^{-1}\right)$. $\mathrm{VO}_{2}$ max for those classified as 'high' was based on data from Australian Rules footballers $\left(60 \mathrm{ml} \mathrm{kg}^{-1}\right.$ $\min ^{-1}$ ) (18). Both trials began with a 5-minute warm up at a self-selected speed that was lower than the assigned running speed for the trial itself. Subjects then completed a 10-minute run at their pre-assigned running speed, followed by a 10-minute passive cool-down in a seated position. The duration of the run was informed by ACSM (17) exercise guidelines for healthy adults which encourages 30-60 minutes of moderate-to-vigorous exercise per day in bouts of 10 minutes or more. This duration was also deemed appropriate as exercise bouts of $\sim 10$ minutes are associated with improved health outcomes and similar health benefits to exercise bouts $>10$ minutes $(19,20)$. Additionally, previous research has implemented similar exercise durations to examine the impact of WR on physiological and perceptual outcomes $(9$, 11).

\subsection{Variables and Measurements}

Physiological responses were measured via $\mathrm{HR}$ and $\mathrm{VO}_{2}$. $\mathrm{HR}$ (beats per minutes; bpm) was measured using a chest strap HR monitor (Polar Electro Oy, KY, Finland). Gas exchange variables including $\mathrm{VO}_{2}(\mathrm{ml}$ $\mathrm{kg}^{1} \mathrm{~min}^{-1}$ ) were measured using a metabolic measurement system (Metalyzer 3B, Cortex Medical, Leipzig, Germany) which was calibrated prior to each testing session with a known composition of ambient air $\left(\mathrm{O}_{2}: 20.93 \%\right.$ and $\mathrm{CO}_{2}: 0.03 \%$ ); this system has previously been shown as reliable (reliability coefficients for $\mathrm{VO}_{2}, \mathrm{VCO}_{2}$ and $\mathrm{VE}=0.969,0.964$ and 0.953 , respectively) (21).

Participants wore a size-appropriate face mask connected to the metabolic measurement system via an air flow line, allowing them to breathe freely through their mouth and nose. HR was measured continuously and $\mathrm{VO}_{2}$ data were recorded breath-by-breath for the duration of the 10-minute run and 10minute passive cool-down. HR steady-state has been reported to occur within 2-4 minutes of exercise at constant sub-maximal work rates $(22,23)$. Thus, to sufficiently achieve the aims of this study, the final five minutes of the running test were analysed, and the first five minutes of the cool-down were examined

for consistency purposes. Perceptual responses were measured via rate of perceived exertion (RPE) using Borg's 6-20 RPE scale (24) every 2.5 minutes during the running portion of the test.

\subsection{Statistical analysis}

All statistical analyses were completed using IBM SPSS Statistics for Windows (version 25.0; IBM Corp., Armonk, N.Y., USA). $\mathrm{HR}$ and $\mathrm{VO}_{2}$ data were smoothed to 30 second intervals to de-emphasise recorded breath-to-breath variations. HR monitor malfunctions were experienced during five separate 30 -second intervals resulting in obvious errors; these data points were subsequently removed before analysis. Assumption testing showed not all variables met parametric assumptions for normality or variance. Given this outcome, and the size of the data sample examined, descriptive statistics (median and 
interquartile range) and percentage changes were calculated for all variables during the run and cooldown portions of the trials. Individual $\mathrm{VO}_{2}$ and $\mathrm{HR}$ responses during the run and cool-down were also examined to understand inter-individual variability. Wilcoxon Signed-Rank Tests were used to compare descriptive statistics between control and experimental trials for all variables, with effect sizes calculated using the following equation;

$r=\frac{Z}{\sqrt{N}}$

Key: r: Effect size, Z: Standardized z-score, N: Total number of observations

Effect sizes were categorised using the following scale; $<0.2=$ trivial effect, $0.2-0.5=$ small effect, $0.5-0.8$ $=$ moderate effect, $>0.8=$ large effect (25). Friedman's test was used to identify significant differences between control and experimental trials over time (trial $\mathrm{x}$ time). Statistical significance was set at $p<0.05$.

\section{Results}

\subsection{Collective responses during exercise and passive recovery}

Physiological responses to exercise for control and experimental trials are presented in Figure 2. Overall, $\mathrm{VO}_{2}$ was $4.5 \%$ higher in experimental trials (41.9 [5.7]) compared to control trials (40.1 [5.8]) with only a small effect $(r=0.24)$. In contrast, HR was slightly lower $(0.4 \%)$ in control trials (161.5 [25.5]) compared to experimental trials (160.9 [24.7]) with a trivial effect $(r=-0.05)$. RPE was $7.7 \%$ higher in the experimental trials (14.0 [3.0]) when compared to control trials (13.0 [3.0]) with a small effect $(r=0.32)$. No statistically significant differences were observed for $\mathrm{VO}_{2}, \mathrm{HR}$ or RPE between experimental and control trials (all $p>$ 0.05).

Physiological responses elicited during post-exercise passive recovery for control and experimental trials can be viewed in Figure 3. Overall, $\mathrm{VO}_{2}$ was $4.7 \%$ lower in experimental trials (8.2 [2.4]) compared to control trials (8.6 [2.1]) with a small effect $(r=-0.32)$. Similar results were seen for HR with experimental trials (95.8 [27.5]) being 4.3\% lower compared to control trials (100.1 [30.8]) with only a small effect ( $r=$ -0.27). A significant effect of time was identified for $\mathrm{VO}_{2}$ and $\mathrm{HR}(p<0.05)$ post-exercise, however no significant differences were observed for $\mathrm{VO}_{2}$ or $\mathrm{HR}$ between experimental and control trials (all $p>0.05$ ).

\subsection{Individual responses during exercise and passive recovery}

Individual physiological responses to exercise in control and experimental trials are presented in Figure 4 (A). $\mathrm{VO}_{2}$ was higher in experimental than control trials for 10 subjects (range: $0.4-12.0 \%$ higher) and lower in experimental than control trials for five subjects (range: 1.9-3.8\% lower). HR was higher in experimental 
than control trials for six subjects (range: $0.4-6.0 \%$ higher) and lower in experimental than control trials for nine subjects (range: $0.3-5.5 \%$ lower).

Individual physiological responses elicited during post-exercise passive recovery for control and experimental trials are also presented in Figure 4 (B). Results showed that $\mathrm{VO}_{2}$ was higher in experimental than control trials for ten subjects (range: $4.4-33.4 \%$ higher) and lower in experimental than control trials for five subjects (range: $1.2-16.4 \%$ lower). Individual responses also showed that HR was higher in experimental than control trials for five subjects (range: 1.0-17.2\% higher) and lower in experimental than control trials for nine subjects (range: 2.2-12.6\% lower). One subject experienced no change in HR between control and experimental trials.

\section{Discussion}

\subsection{Responses during exercise}

During steady-state running, $\mathrm{VO}_{2}$ and $\mathrm{RPE}$ were higher (by $4.5 \%$ and $7.7 \%$, respectively) and $\mathrm{HR}$ was $0.4 \%$ lower during experimental trials wearing the resistance garment than during control trials wearing a standard pair of exercise shorts, although the magnitudes of difference were trivial to small, and not statistically significant. There was large inter-individual variability; $\mathrm{VO}_{2}$ was higher in experimental trials for 10 of the 15 subjects (range: $0.4-12.0 \%$ higher compared to control trials) and HR was lower in

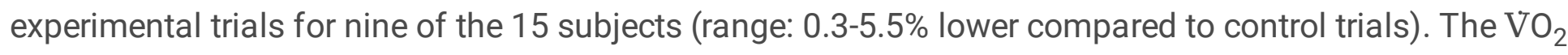
response to exercise in this study is consistent with previously reported findings that lower limb loading increases the metabolic cost of exercise when compared to exercising in unloaded conditions (9-11). Whilst the increase in $\mathrm{VO}_{2}$ during the experimental trials was of small effect in the current study $(r=$ $0.24)$, the magnitude of this increase $(4.5 \%)$ is greater than that reported by Martin (9) who attached 0.5 $\mathrm{kg}$ weights to the thighs and feet (1.7\% and $3.3 \%$ respectively) and attached $1.0 \mathrm{~kg}$ weights to the thighs (3.5\%) during continuous running. Others have reported similar increases in $\mathrm{VO}_{2}$ to the present study during submaximal running; Claremont and Hall (10) reported a $4.5 \%$ increase with the use of $0.45 \mathrm{~kg}$ ankle weights and Field et al. (11) reported an increase of $4.3 \%$ with the use of weighted pouches stitched into compression shorts equating to $3 \% \mathrm{BM}$ resistance. Comparable increases in $\mathrm{VO}_{2}$ between the current study and previous research indicates the method of application of load to the garment in the current study (i.e. resistive bands woven into the material of the resistance garment) has similar potential to increase the metabolic demand as previously reported methods, with the added benefit of improved design features. However, caution should be used when generalising these findings as five of the 15 subjects in the current study recorded a lower $\mathrm{VO}_{2}$ in experimental trials (range: 1.9-3.8\% lower compared to control trials), suggesting that the current WR garment may not influence the metabolic demand of running for everyone equally. Likely explanations for this may relate to differences in body morphology or the approach used to prescribe exercise intensity in the current study (i.e. assigning running speed based on physical activity levels vs. using a relative percentage of each subject's $\mathrm{VO}_{2} \mathrm{max}$ ). Nevertheless, there is a trend to suggest that the magnitude of applied resistance provided by the garment in the current 
study $(1-3 \% \mathrm{BM})$ is likely sufficient to provide a stimulus that increases $\mathrm{VO}_{2}$ during a single bout of exercise. This finding supports those of Field et al. (11) who also observed $1.7 \%, 2.4 \%$ and $4.3 \%$ increases in $\mathrm{VO}_{2}$ when $1 \%, 2 \%$ and $3 \% \mathrm{BM}$ resistance, respectively, were applied via lower limb loading during running. However, Field et al. (11) also observed $5.4 \%$ and $8.1 \%$ increases in $\mathrm{VO}_{2}$ when $4 \%$ and $5 \% \mathrm{BM}$ resistance was applied, respectively, suggesting that $\mathrm{VO}_{2}$ may indeed continue to increase with increasing WR load. Therefore, future research is warranted to investigate an "upper limit" of WR that results in an increase in $\mathrm{VO}_{2}$ without a concomitant decrease in movement quality. Future research should also extend on the present investigation and assess whether the current WR garment elicits a similar $\mathrm{VO}_{2}$ response during exercise exceeding 10 minutes in duration and/or exceeding submaximal intensities that are individualized to each subject's $\mathrm{VO}_{2}$ max.

The perceived impact of the WR garment was insignificant, as shown by only a slightly higher RPE during the experimental (14 [3.0]) compared to the control trial (13 [3.0]). Two previous studies have examined the perceived impact of WR and external loading on the user during running and walking; both studies reported considerable increases in RPE when the \% of BM resistance was increased using weighted back packs (8) and compression shorts (11). Key methodological differences may explain, at least in part, why the present results do not align with those previously reported. The current study only assessed 1-3\% BM resistance whereas Simpson et al. (8) assessed 20\%, 30\% and 40\% BM resistance. In the current study, subjects completed $2 \times 10$-minute steady-state runs with five days between trials whereas in the study by Field et al. (11), subjects completed $6 \times 8$-minute submaximal runs over two separate testing sessions with only 2-3 days recovery between sessions. Additionally, it is unclear whether higher RPE scores in the Simpson et al. (8) study were the result of increases in BM resistance or a reflection of discomfort experienced by the subjects given the high discomfort ratings reported in the shoulders, neck, upper back, lower back, hips, thighs and lower legs. Although results from the current study do not support those from previous literature, the differences across studies highlights the need to further examine the perceived impact of WR during exercise using a variety of WR methods and applied magnitudes of resistance. Additionally, future research should also consider other perceptual measures that the current study did not explore such as comfort, to determine the practicality of WR methods more holistically.

In contrast to $\mathrm{VO}_{2}$, there was no difference in $\mathrm{HR}$ responses to exercise between the experimental and control trials. Indeed, HR was actually $0.4 \%$ lower in the experimental compared to control trials, although the effect size was trivial $(r=-0.05)$. This finding is unique when compared to previous literature as a variety of studies using lower limb loading (9-11) and upper body loading (8) noted an increase in HR when incorporating WR during exercise. Claremont and Hall (10) observed a $2.7 \%$ increase in HR when $0.45 \mathrm{~kg}$ ankle weights were used during continuous running. Martin (9) noted HR increases of $0.5 \%$ and $1.6 \%$ when $0.50 \mathrm{~kg}$ lead shot weights were attached to the thigh and feet respectively, and $1.4 \%$ and $3.4 \%$ $\mathrm{HR}$ increases with $1.0 \mathrm{~kg}$ lead shot weights attached to the aforementioned body segments. Field et al. (11) also reported increases in $\mathrm{HR}(0.4 \%, 1.5 \%$ and $1.8 \%)$ when $1 \%, 2 \%$ and $3 \%$ BM resistance was applied, respectively, via weighted pouches stitched onto compression shorts during sub-maximal running. Differences in the methods of WR used, and the loads applied between the current study and 
others may explain, at least in part, why different HR responses were seen. The general design of the garment in the present study and use of resistive bands may not provide consistent and/or sufficient load or resistance to influence HR during locomotion when compared to the addition of weighted pouches or lead shots used in previous research $(9,11)$. However, further studies incorporating biomechanical analyses are needed before making conclusions regarding loading over a gait cycle. Additionally, the garments were not designed to specifically fit each subject's individual body shape and size which may explain, at least in part, why nine of the fifteen subjects in the present study recorded a lower HR overall during experimental than control trials (range: $0.3-5.5 \%$ lower). The magnitude of HR increases in previous literature is lower than the magnitude of increase seen for $\mathrm{VO}_{2}$ (9-11), and this is consistent with the present study. This may suggest that $\mathrm{VO}_{2}$ is more sensitive to identifying a change in physiological load with WR, and therefore HR may have a higher threshold for detecting additional load applied in the form of WR garments. Future research examining the different detection limits of $\mathrm{VO}_{2}$ and $\mathrm{HR}$ over a range of resistances is needed to confirm this observation and identify their respective sensitivity thresholds.

\subsection{Responses during passive recovery}

During passive recovery following steady-state running, $\mathrm{VO}_{2}$ and $\mathrm{HR}$ were lower (by $4.7 \%$ and $4.3 \%$, respectively) during the experimental than the control trial and the magnitudes of difference were small and not statistically significant. $\mathrm{VO}_{2}$ was higher in experimental comparted to control trials for ten of the fifteen subjects (range: 4.4-33.4\% higher) and HR was lower in experimental compared to control trials for nine of the fifteen subjects (range: $2.2-12.6 \%$ lower), indicating large inter-individual variability in responses. This variation in individual responses highlights the need for caution when generalising the main findings of the present study, especially given the small sample size. Nevertheless, this finding might hold some practical significance, since even small improvements in recovery might be valuable for exercise involving repeated efforts, such as high-intensity interval training where the intensity of the subsequent exercise bout is influenced by the preceding recovery bout $(26,27)$. This finding is unique given the current study is the first to analyse the impact of lower limb WR on acute recovery following exercise as well as assessing a unique application of WR wholly contained within a garment and not using external weights. These findings provide a foundation for future research to investigate the influence of exercise intensity, duration and nature (continuous vs. intermittent) on recovery whilst wearing WR garments. The design of the garment used in the present study may explain, at least in part, why a small improvement in physiological recovery was seen during the experimental trial. The overall design and materials used ensured the garment smoothly conformed to the user's individualized body shape creating a tight fit, similarly to a compression garment. Recent research has shown whole-body compression garments significantly reduced HR following incremental running tests in untrained subjects when compared to non-compression garments $(28,29)$. When applied to garments, compression is proposed to improve recovery by mitigating the physiological strain of exercise via increased localized blood flow and provision of oxygen and improved venous return to remove metabolites following exercise (14). While these mechanisms may partly explain the small reductions in $\mathrm{VO}_{2}$ and $\mathrm{HR}$ during passive 
recovery post-exercise, results should be interpreted with caution when determining the capacity of the garment to promote post-exercise recovery. These findings support the need for future research to further explore the impact of WR on recovery and the physiological mechanisms responsible. The measurement of additional metabolic variables such as blood lactate concentration may also be beneficial when exploring the extent to which WR increases the physiological demand of exercise, and its impact on postexercise recovery.

\section{Conclusions}

Although the effects were small, the novel WR garment increased $\mathrm{VO}_{2}$ and RPE during steady-state running, and reduced $\mathrm{VO}_{2}$ and $\mathrm{HR}$ during passive recovery, compared to control. These findings provide proof of concept and suggest that this garment design may be effective as a mechanism to increase training stimulus during running and as a recovery aid post-exercise, in recreationally-active men.

\section{Abbreviations}

ACSM American College of Sports Medicine

BM Body Mass

BMI Body Mass Index

$\mathrm{CO}_{2}$ Carbon Dioxide

FEA Finite Element Analysis

HR Heart Rate

$\mathrm{O}_{2}$ Oxygen

RPE Rate of Perceived Exertion

$\mathrm{VCO}_{2}$ Volume of Exhaled Cardon Dioxide

VE Ventilation volume

$\mathrm{VO}_{2}$ Oxygen Consumption

$\mathrm{VO}_{2}$ max Maximal Oxygen Consumption

WR Wearable Resistance

\section{Declarations}




\section{Ethics approval and consent to participate}

Ethics approval was obtained from the Deakin University Human Ethics Advisory Group (Health) prior to participant recruitment (HEAG-H 131_2019). All subjects provided written informed consent before participating. All procedures were carried out in accordance with relevant guidelines and regulations.

\section{Consent for publication}

Not applicable.

\section{Availability of data and materials}

The datasets used and/or analyzed during the current study are available from the corresponding author on reasonable request.

\section{Competing interests}

The authors declare that the research was conducted in the absence of any commercial or financial relationships that could be construed as a potential conflict of interest. The authors declare that they have no competing interests.

\section{Funding}

Funding for this project was provided from 6 0'clock Enterprises. 6 0'clock Enterprises had no role in the design of the study, data collection, data analyses, interpretation of data or in writing the manuscript.

\section{Author's contributions}

$\mathrm{SH}$ - Assisted with the development of the study design, oversaw data collection, and was a major contributor in critically revising this manuscript

IDD - Assisted with the development of the study design, was a major contributor in participant data collection and statistical analyses and was a major contributor in writing this manuscript.

PC - Assisted with the development of the study design, assisted in the ideation, design and development of the wearable resistance garment, and was a major contributor in critically revising this manuscript.

\section{Acknowledgements}

The authors would like to acknowledge the support and funding from 6 O'clock Enterprises and the infrastructure resources provided by the Schools of Engineering and Exercise and Nutrition Sciences at Deakin University. The authors would also like to thank the participants for volunteering their time and effort for the project.

\section{References}


1. Kraemer WJ, Ratamess NA, French DN. Resistance training for health and performance. Curr Sports Med Rep. 2002;1(3):165-71.

2. McNicol AJ, O'Brien BJ, Paton CD, Knez WL. The effects of increased absolute training intensity on adaptations to endurance exercise training. J Sci Med Sport. 2009;12:485-9.

3. Hrysomallis $\mathrm{C}$. The effectiveness of resisted movement training on sprinting and jumping performance. J Strength Cond Res. 2012;26(1):299-306.

4. Macadam P, Cronin JB, Simperingham KD. The effects of wearable resistance training on metabolic, kinematic and kinetic variables during walking, running, sprint running and jumping: A systematic review. Sports Med. 2017;47(5):887-906.

5. Macadam P, Cronin JB, Uthoff AM, Feser EH. The effects of different wearable resistance placements on sprint-running performance: A review and practical applications. Strength Cond J. 2019;41(3):7996.

6. Cooke CB, McDonagh MJN, Nevill AM, Davies CTM. Effects of load on oxygen intake in trained boys and men during treadmill running. J Appl Physiol. 1991;71(4):1237-44.

7. Puthoff ML, Darter BJ, Nielsen DH, Yack HJ. The effect of weighted vest walking on metabolic responses and ground reaction forces. Med Sci Sports Exerc. 2006;38(4):746-52.

8. Simpson KM, Munro BJ, Steele JR. Effect of load mass on posture, heart rate and subjective responses of recreational female hikers to prolonged load carriage. Appl Ergon. 2011;42:403-10.

9. Martin PE. Mechanical and physiological responses to lower extremity loading during running. Med Sci Sports Exerc. 1985;17(4):427-33.

10. Claremont AD, Hall SJ. Effects of extremity loading upon energy expenditure and running mechanics. Med Sci Sports Exerc. 1988;20(2):167-71.

11. Field AP, Gill N, Macadam P, Plews D. Acute metabolic changes with thigh-positioned wearable resistances during submaximal running in endurance-trained runners. Sports. 2019;7(8):187-99.

12. Rusko $\mathrm{H}$, Bosco $\mathrm{C}$. Metabolic response of endurance athletes to training with added load. Eur J Appl Physiol Occup Physiol. 1987;56(4):412-8.

13. Couture GA, Simperingham KD, Cronin JB, Lorimer AV, Kilding AE, Macadam P. Effects of upper and lower body wearable resistance on spatio-temporal and kinetic parameters during running. Sports Biomech. 2018;19(5):633-51.

14. MacRae BA, Cotter JD, Laing RM. Compression garments and exercise. Sports Med. 2011;41(10):815-43.

15. Collins P, Harb C, Mikelsons B. Resistance garments having integral seamless resistive zones. 2016. Contract No.: 2016205887.

16. Jones AM, Doust JH. A $1 \%$ treadmill grade most accurately reflects the energetic cost of outdoor running. J Sports Sci. 1996;14(4):321-7.

17. American College of Sports Medicine. ACSM's guidelines for exercise testing and prescription. 10th ed. Philadelphia: Wolters Kluwer Health; 2018. 
18. Buttifant D. Physiological and performance characteristics of Australian Football League players. J Sports Sci. 1999;17:808-9.

19. Jakicic J, Kraus W, Powell K, Campbell W, Janz K, Troiano R, et al. Association between bout duration of physical activity and health: Systematic review. Med Sci Sports Exerc. 2019;51:1213-9.

20. Murphy M, Lahart I, Carlin A, Murtagh E. The effects of continuous compared to accumulated exercise on health: A Meta-analytic review. Sports Med. 2019;49:1585-607.

21. Meyer T, Georg T, Becker C, Kindermann W. Reliability of gas exchange measurements from two different spiroergometry systems. Int J Sports Med. 2001;22(8):593-7.

22. Koike A, Yajima T, Adachi H, Shimizu N, Kano H, Sugimoto K, et al. Evaluation of exercise capacity using submaximal exercise at a constant work rate in patients with cardiovascular disease. Circulation. 1995;91(6):1719-24.

23. Whipp BJ. Rate constant for the kinetics of oxygen uptake during light exercise. J Appl Physiol. 1971;30(2):261-3.

24. Borg GA. Psychophysical bases of perceived exertion. Med Sci Sports Exerc. 1982;14(5):377-81.

25. Cohen J. Statistical power analysis for the behavioural sciences. 2nd ed. New Jersey: Lawrence Erlbaum; 1988.

26. Schoenmakers PPJM, Hettinga FJ, Reed KE. The moderating role of recovery durations in highintensity interval-training protocols. Int J Sports Physiol Perform. 2019;14(6):859-67.

27. Schoenmakers PPJM, Reed KE. The effects of recovery duration on physiological and perceptual responses of trained runners during four self-paced HIIT sessions. J Sci Med Sport. 2019;22(4):4626.

28. Nguyen TNL, Eager D, Nguyen HT. Effect of compression garments on cardiovascular function during recovery phase. Conf Proc IEEE Eng Med Biol Soc. 2018:2869-72.

29. Nguyen TNL, Eager D, Nguyen HT. The relationship between compression garments and electrocardiogram signals during exercise and recovery phase. Biomed Eng Online. 2019;18:27-36.

\section{Figures}



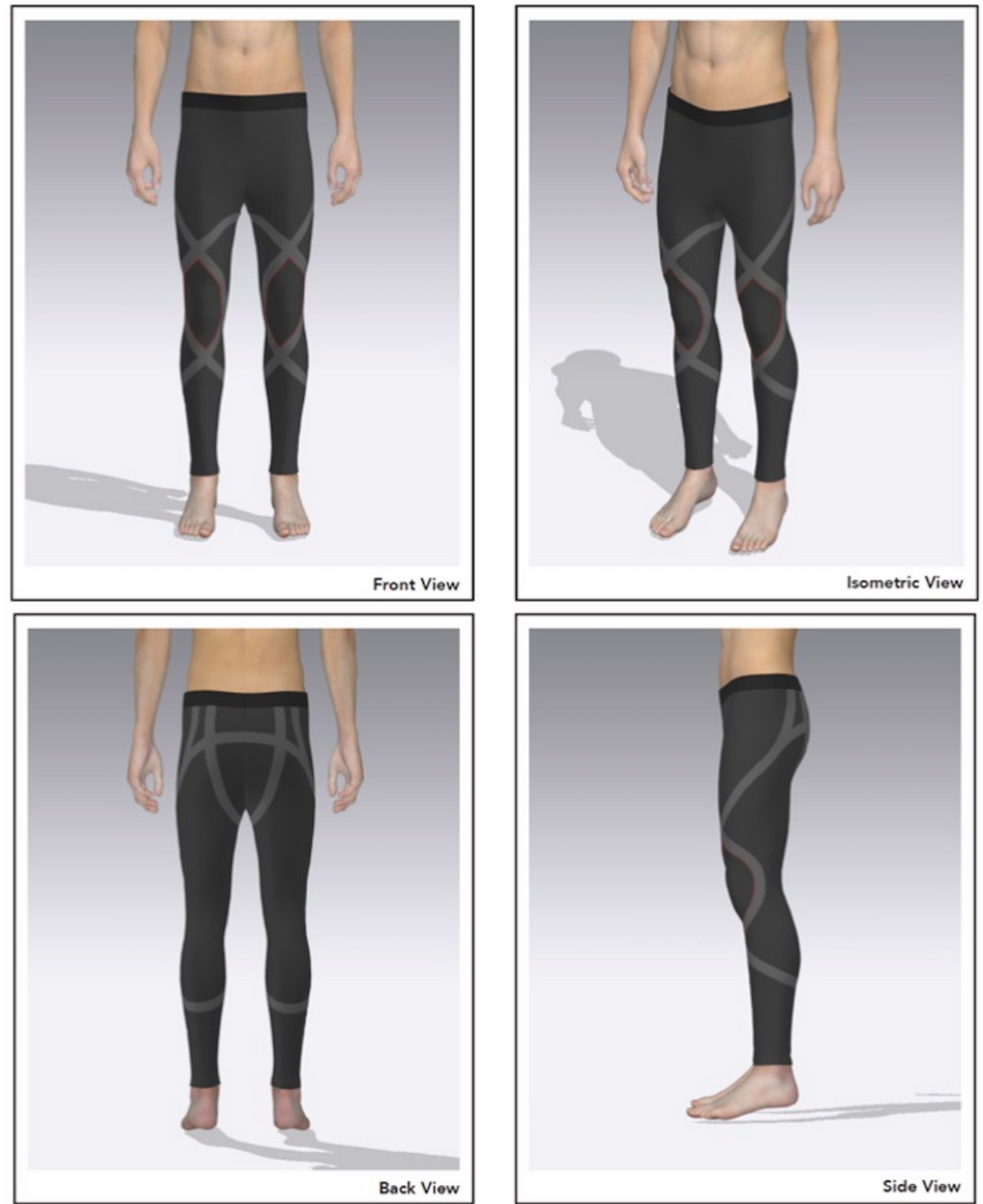

Figure 1

General design of the novel wearable resistance garment. 

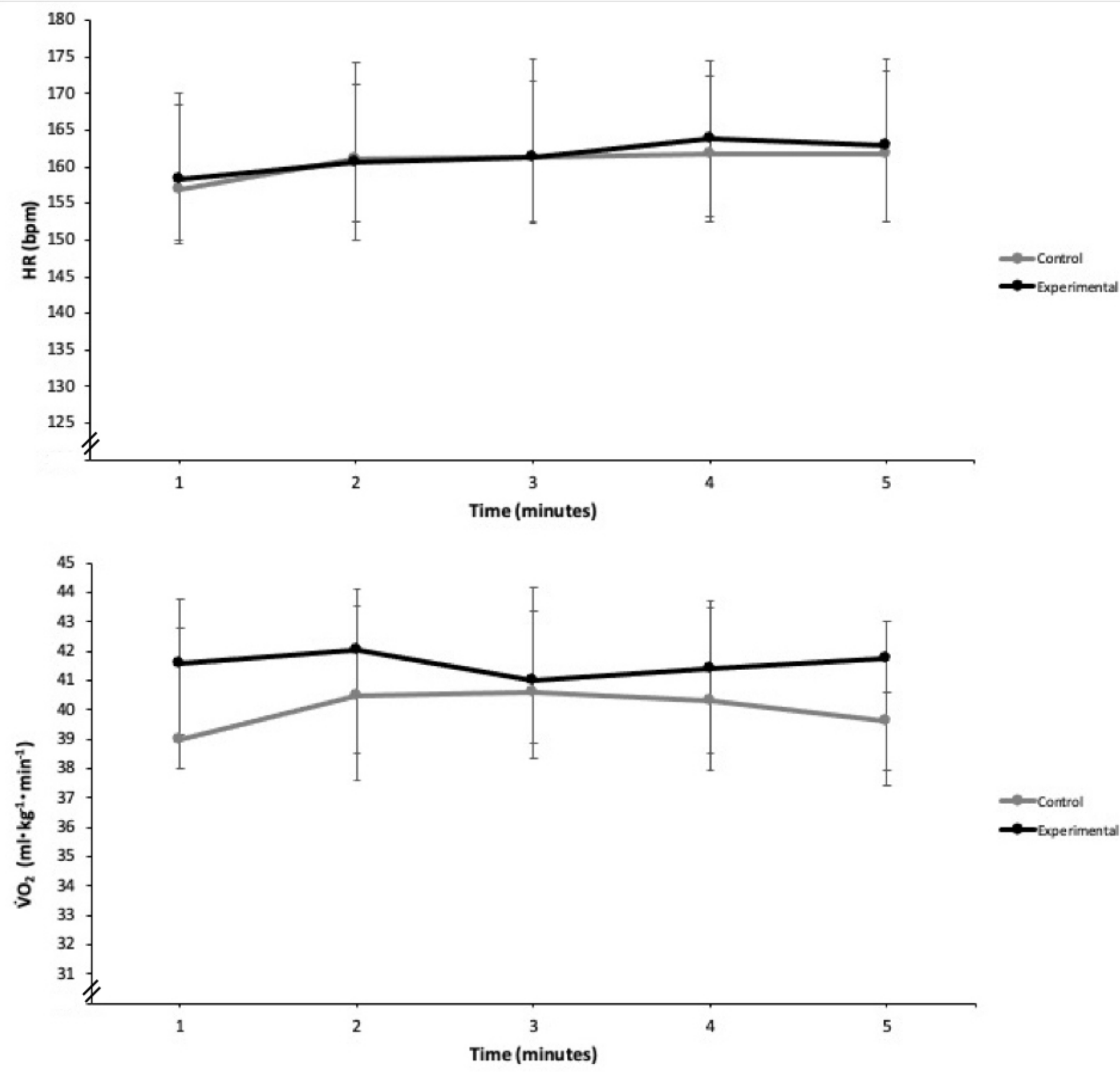

Figure 2

Physiological responses during five minutes steady-state running in control and experimental trials $(\mathrm{n}=$ 15). Data presented as median with interquartile range error bars. V02: oxygen consumption; $\mathrm{mL}$ : millilitre; kg: kilogram; min: minute; HR: heart rate; bpm: beats per minute. 

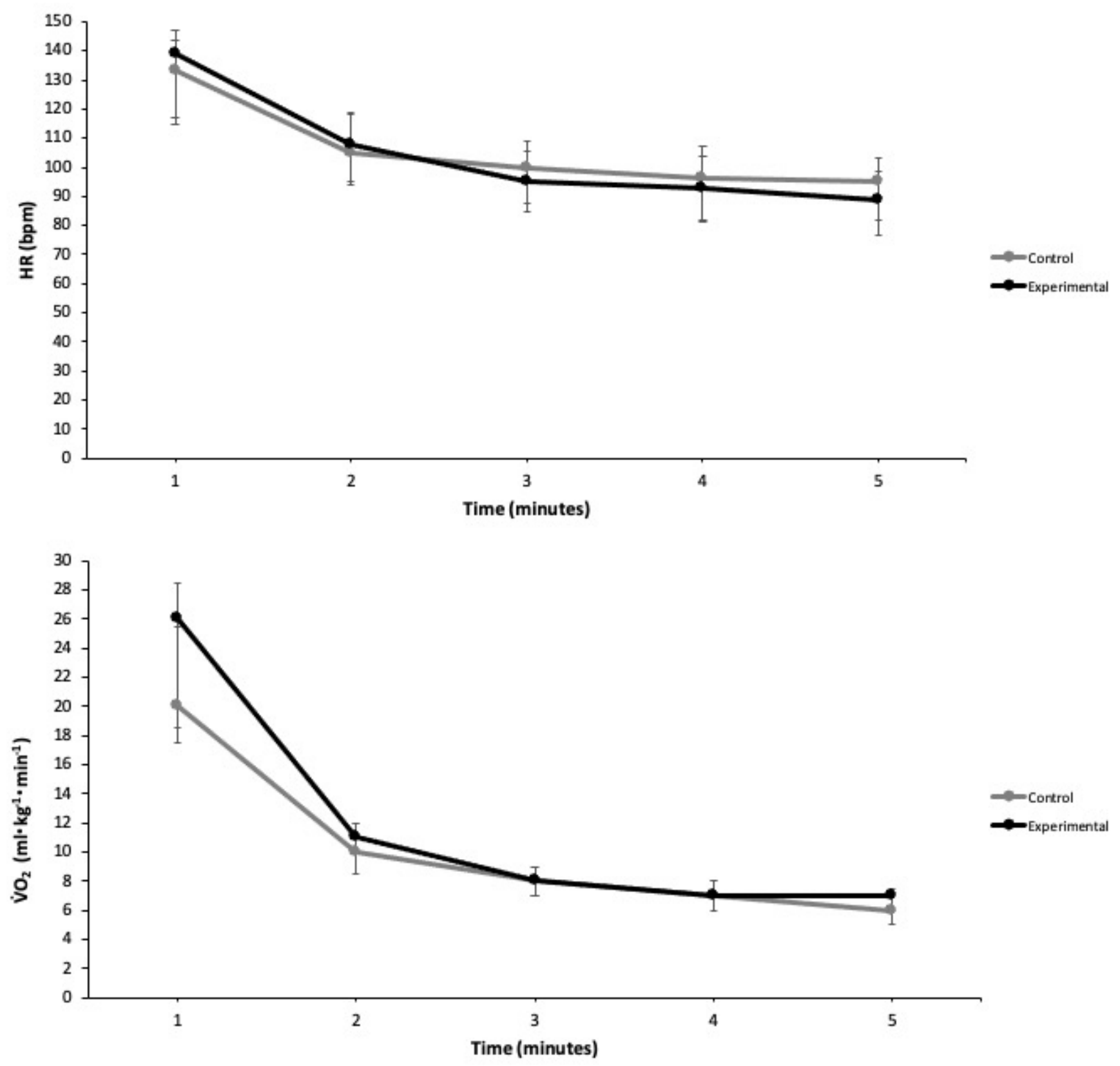

\section{Figure 3}

Physiological responses during five minutes post-exercise recovery in control and experimental trials ( $\mathrm{n}=$ 15). Data presented as median with interquartile range error bars. V02: oxygen consumption; $\mathrm{mL}$ : millilitre; kg: kilogram; min: minute; HR: heart rate; bpm: beats per minute. 

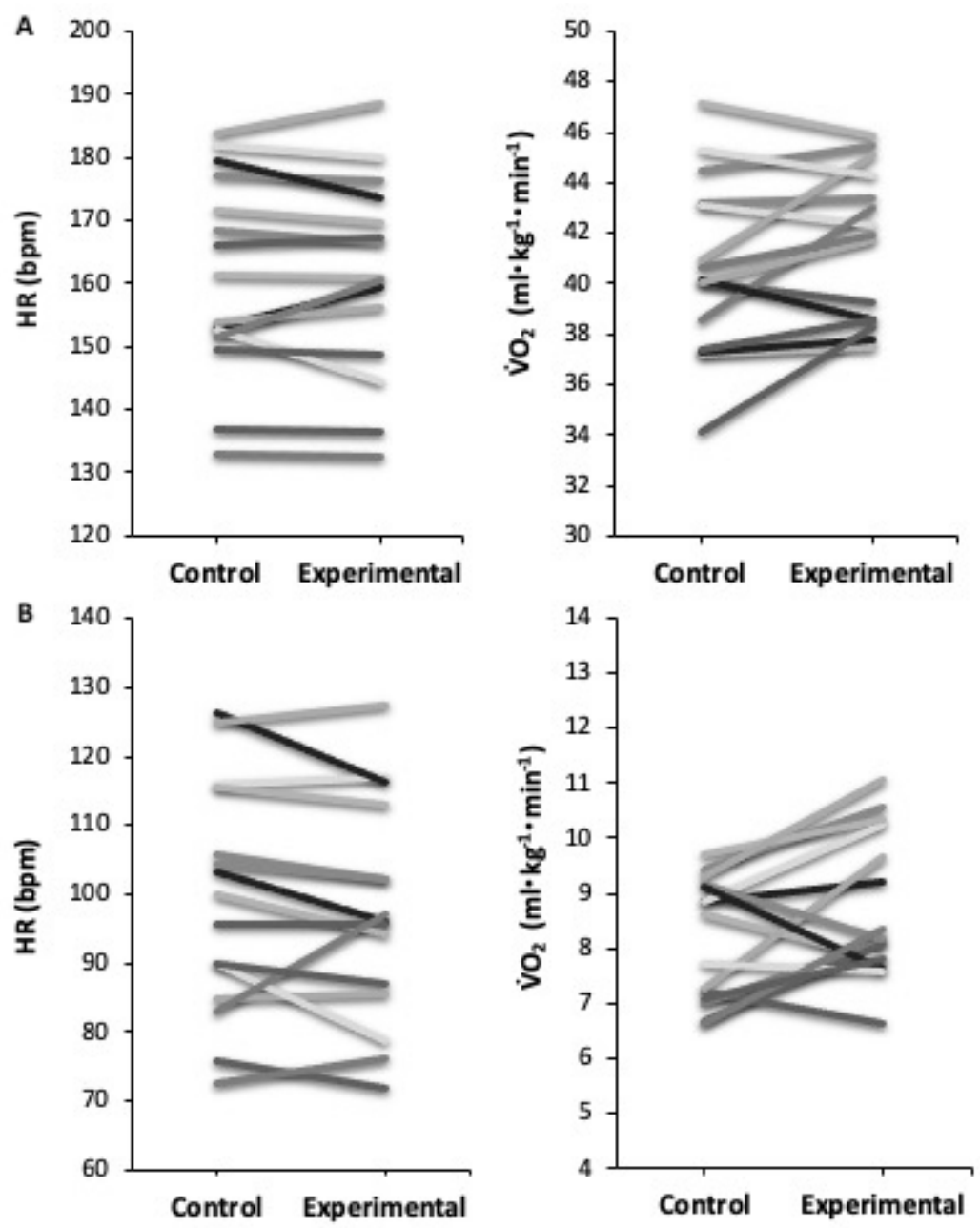

Figure 4

Individual responses during (A) five minutes steady-state running and (B) post-exercise recovery $(n=15)$. Results for control and experimental trials are presented as median per subject. V02: oxygen consumption; $\mathrm{mL}$ : millilitre; kg: kilogram; min: minute; HR: heart rate; bpm: beats per minute. 